\title{
PRODUCTIVIDAD Y CALIDAD DE FORRAJE DE HÍBRIDOS TRILINEALES DE MAÍZ PARA VALLES ALTOS DE MÉXICO
}

\author{
PRODUCTIVITY AND QUALITY OF FORAGE OF THREE-WAY MAIZE \\ HYBRIDS FOR THE HIGH VALLEYS OF MEXICO
}

\author{
Job Zaragoza-Esparza', María Fernanda Medina-Fernández', Margarita Tadeo- \\ Robledo ${ }^{1}$, Alejandro Espinosa-Calderón ${ }^{2}$, Consuelo López-López ${ }^{1}$ *, Enrique \\ Canales-Islas ${ }^{3}$, Arturo Chávez-Gordillo ${ }^{1}$ y Homero Alonso-Sánchez ${ }^{1}$
}

\begin{abstract}
'Universidad Nacional Autónoma de México, Facultad de Estudios Superiores Cuautitlán, Ingeniería Agrícola, Cuautitlán Izcalli, Estado de México, México. ${ }^{2}$ Instituto Nacional de Investigaciones Forestales, Agrícolas y Pecuarias (INIFAP), Campo Experimental Valle de México, Coatlinchán, Texcoco, Estado de México, México. ${ }^{3}$ INIFAP, Campo Experimental Santiago Ixcuintla, Santiago Ixcuintla, Nayarit, México.
\end{abstract}

\section{RESUMEN}

La producción de maíces forrajeros con altos rendimientos y buena calidad es una solución favorable para contrarrestar la falta de alimentos para el ganado y altos costos de producción de forraje, debido a que el maíz utilizado como ensilado constituye del 30 al $40 \%$ de la ración diaria utilizada para la alimentación de bovinos productores de leche. El objetivo del presente estudio fue determinar el rendimiento de forraje y valor nutricional de nueve híbridos de maíz e identificar aquellos que puedan constituir una alternativa para los productores de bovinos de leche de los Valles Altos de México. Los híbridos evaluados fueron H-47 AE, H-49 AE, H-51 AE, H-53 AE, H 61 R, Tlaoli Puma, Tsíri Puma y Atziri Puma, así como un material experimental. El experimento se estableció en tres ambientes: una fecha de siembra en la FES CuautitlánUNAM y dos en el Campo Experimental Valle de México (CEVAMEX) del INIFAP. Se utilizó un diseño experimental de bloques completos al azar con tres repeticiones, bajo un arreglo factorial que consideró como factores a los ambientes y a los genotipos. Tlaoli Puma y H-49 AE presentaron características productivas deseables con respecto a los otros híbridos evaluados, ya que tuvieron los mayores rendimientos de materia seca (23.1 y $21.0 \mathrm{t} \mathrm{ha}^{-1}$, respectivamente), digestibilidad in vitro de la materia seca (67.0 a $69.8 \%$ ) y proteína cruda (8.1 y $8.4 \%$ ). La cosecha en la FES Cuautitlán-UNAM a los 125 días de desarrollo produjo mayores rendimientos de materia verde y seca, menor porcentaje de proteína cruda y menores contenidos de fibra detergente ácido (FDA) con respecto a los ambientes del CEVAMEX. Los híbridos Tlaoli Puma y H-49 AE constituyen una alternativa para los productores de ganado bovino de Valles Altos de México.

Palabras clave: Maíz forrajero, digestibilidad in vitro, fibra detergente ácido, fibra detergente neutro, rendimiento de forraje.

\section{SUMMARY}

Production of fodder maize with high yields and good quality is a favorable solution to counteract the lack of feed and high costs of forage production, since maize used as silage constitutes 30 to $40 \%$ of the daily ration used for feeding dairy cattle. The objective of this study was to determine the forage yield and nutritional value of nine maize hybrids and identify those that could constitute an alternative for dairy cattle producers in the High Valleys of Mexico. The hybrids evaluated were $\mathrm{H}-47 \mathrm{AE}, \mathrm{H}-49$ AE, $\mathrm{H}-51$ AE, $\mathrm{H}-53 \mathrm{AE}, \mathrm{H}$ 61 R, Tlaoli Puma, Tsíri Puma and Atziri Puma, along with an experimental material. The experiment was established in three environments: one planting date at FES Cuautitlan-UNAM and two at the High Valleys Experiment
Station (CEVAMEX) of INIFAP. A complete randomized blocks experimental design with three replications was used under a factorial arrangement that considered the environments and genotypes as factors. Tlaoli Puma and H-49 AE presented desirable productive characteristics compared to the other hybrids evaluated, since they produced the highest dry matter yields (23.1 and $21.0 \mathrm{t} \mathrm{ha}^{-1}$, respectively), in vitro dry matter digestibility (67.0 and 69.8 $\%$ ) and crude protein (8.1 and $8.4 \%$ ). The harvest at FES Cuautitlán-UNAM at 125 days of development, produced higher yields in fresh and dry matter, lower percentage of crude protein and lower contents of acid detergent fiber (FDA) compared to the CEVAMEX environments. The Tlaoli Puma and H-49 AE hybrids are an alternative for cattle producers of the High Valleys of Mexico.

Index words: Acid detergent fiber, forage maize, forage yield, in vitro digestibility, neutral detergent fiber.

\section{INTRODUCCIÓN}

El maíz forrajero es un cultivo de gran importancia para la ganadería debido a que se puede suministrar en fresco, como ensilado o como rastrojo. Con el ensilaje, grandes cantidades de forraje pueden ser conservadas rápidamente, la cosecha y el proceso son menos dependientes del clima que el henificado (Grant y Adesongant, 2018); asimismo, permite disponer de alimento de buena calidad durante el invierno, debido a su alto valor energético, considerando que es una época crítica de escasez de forraje; además, aporta de 8 a $9 \%$ de proteína (Zaragoza -Esparza et al., 2019) y fibra digestible (70\%), lo que lo hace un componente importante de una ración alimenticia para el ganado, sobre todo el lechero. En las cuencas lecheras de México, el ensilado de maíz se utiliza en la alimentación de este ganado y llega a constituir del 30 al 40 \% de la ración diaria para vacas en producción (González et al., 2005). Algunas ventajas que presenta el maíz para producción de ensilado en comparación con otras especies son un elevado rendimiento de materia verde, de hasta $82 \mathrm{t} \mathrm{ha-1}^{-1}$ (Rivas, 2018), rápido crecimiento, alto contenido de energía $\left(1.52 \pm 0.05 \mathrm{Mcal}^{\mathrm{kg}}{ }^{-1}\right)$ (Núñez, 2003), calidad uniforme, 
resistencia a enfermedades foliares, se requiere de una sola cosecha y produce un rendimiento de materia seca dos veces superior al de otros cultivos (Martin et al., 2017) tales como avena, triticale, ceba y trigo (Núñez, 2015).

La selección de híbridos de maíz para forraje se basa en la producción de materia seca por unidad de superficie y variables de calidad nutricional. En relación con esto último, las evaluaciones que se han realizado consideran variables de composición química como proteína cruda, fibra detergente ácido, fibra detergente neutro, así como digestibilidad de la fibra detergente neutro, contenido de carbohidratos no estructurales y contenido de almidón, en adición a su valor de energía neta de lactancia (Núñez, 2015).

La producción de maíces forrajeros con altos rendimientos, buena calidad y a bajo costo es una solución favorable para contrarrestar la falta de alimentos y altos costos de producción (Rivas et al., 2018); debido a lo anterior, en las zonas de los Valles Altos de México, región ubicada entre los 2200 y 2600 msnm es necesario ofrecer más y mejores variedades para los productores de maíz, por lo que se requiere evaluar híbridos de maíz que se adapten a las condiciones ambientales y que presenten características de rendimiento y calidad de forraje superiores o similares a los híbridos comerciales, con el objetivo de determinar las mejores variedades y ofrecer a los productores una alternativa tecnológica accesible.

Se realizó la presente investigación con el objetivo de determinar el rendimiento de forraje y valor nutricional de nueve híbridos de maíz en tres ambientes e identificar aquellos híbridos que puedan constituir una alternativa para los productores de Valles Altos de México.

\section{MATERIALES Y MÉTODOS}

\section{Sitios experimentales}

La investigación se realizó en el ciclo primavera verano de 2016. Se establecieron tres experimentos, uno en el Centro de Enseñanza Agropecuaria de la Facultad de Estudios Superiores Cuautitlán, Campo 4, de la Universidad Nacional Autónoma de México (FES Cuautitlán, UNAM), ubicada en el municipio de Cuautitlán Izcalli, Estado de México, en las coordenadas geográficas $19^{\circ} 41^{\prime}$ latitud N y $99^{\circ} 11^{\prime}$ longitud O, a una altitud de $2274 \mathrm{msnm}$. El segundo y tercero en el Campo Experimental Valle de México (CEVAMEX) del Instituto Nacional de Investigaciones Forestales, Agrícolas y Pecuarias (INIFAP), ubicado en Santa Lucía de Prías, Coatlinchán, en el municipio de Texcoco a los $19^{\circ} 27^{\prime}$ latitud $\mathrm{N}$ y a $98^{\circ} 51^{\prime}$ de longitud $\mathrm{O}$, a una altitud de $2240 \mathrm{msnm}$, donde se establecieron dos experimentos con diferencia en fecha de cosecha.

\section{Material genético}

Se evaluaron nueve híbridos trilineales de grano blanco: H-47 AE, H-49 AE, H-51 AE y H-53 AE del INIFAP, junto con Tlaoli Puma, Tsíri Puma y Atziri Puma, que fueron desarrollados en la UNAM, así como los híbridos experimentales H 61 R y CSE FESC1.

\section{Diseño y unidad expeimental}

La evaluación se realizó bajo un diseño experimental de bloques completos al azar con tres repeticiones bajo un arreglo factorial que consideró como factores a los ambientes (localidades/fechas de cosecha y a los genotipos (nueve híbridos). La unidad experimental consistió en un surco de $5 \mathrm{~m}$ de largo con una distancia entre surcos de $0.8 \mathrm{~m}$.

\section{Manejo del experimento}

La siembra se realizó el 6 de junio del 2016 en la FES Cuautitlán, UNAM y el 25 de mayo de 2016 en el CEVAMEX. El terreno se preparó de forma convencional; es decir, paso de arado, dos pasos de rastra y surcado; posteriormente, se fertilizó con una dosis de 80-60-00 (N-P-K). La siembra se realizó depositando tres semillas por mata. Al siguiente día se realizó un riego rodado y a los 10 días posteriores un segundo riego; posteriormente, se realizó un aclareo, dejando 32 plantas por unidad experimental para obtener una densidad de población de 80,000 plantas ha-1 ${ }^{-1}$ La cosecha se realizó en tres fechas, correspondiendo cada una a un ambiente; el 9 de octubre; es decir, 126 días después de la siembra (dds) en la FES Cuautitlán UNAM (ambiente FES Cuautitlán, UNAM), mientras que en CEVAMEX se cosechó el 5 y 19 de octubre, a los 134 y 148 dds, respectivamente (ambientes: CEVAMEX1 y CEVAMEX2).

\section{Variables evaluadas}

Se determinó el rendimiento de materia verde (MV) en $\mathrm{t} \mathrm{ha}^{-1}$, a partir de la cosecha de todas las plantas de cada unidad experimental, a una altura de 7 a $10 \mathrm{~cm}$ con respecto al suelo, se pesaron y se utilizó la fórmula siguiente:

Rendimiento de MV $\left(\mathrm{t} \mathrm{ha}^{-1}\right)=\frac{\left(10,000 \mathrm{~m}^{2}\right) \times(\text { Peso fresco, } \mathrm{kg})}{\left(4 \mathrm{~m}^{2}\right)} \times 1000$

El rendimiento de materia seca (MS) en t ha ${ }^{-1}$ se calculó a partir del rendimiento de materia verde y del porcentaje de materia seca utilizando la siguiente fórmula: 
Rendimiento de MS $\left(\mathrm{t} \mathrm{ha}^{-1}\right)=($ Porcentaje de MS $) \times($ Rendimiento de MV $)$ 100

El porcentaje de materia seca se determinó con base en una muestra de forraje fresco de cada unidad experimental, con un peso aproximado de $500 \mathrm{~g}$, se colocó en un horno de convección forzada (Felisa, Modelo FE 293-A, SFELIGNEO S. A. de C.V., Zapopan, Jalisco, México) a $55^{\circ} \mathrm{C}$ hasta que alcanzó peso constante, se pesó y se utilizó la ecuación siguiente:

Porcentaje de materia seca $=$ Peso seco de la muestra $(\mathrm{kg})$

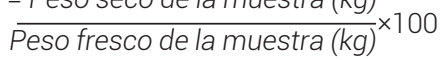

Para determinar el porcentaje de mazorca, de cada parcela experimental se eligieron al azar cinco plantas, a las cuales se les desprendió la mazorca, y juntas se secaron en un horno de convección forzada (Felisa, Modelo FE 293A) a $55^{\circ} \mathrm{C}$ hasta alcanzar un peso constante, se determinó el peso promedio de las mazorcas y se utilizó la siguiente ecuación:

Porcentaje de mazorca $=$ Peso seco promedio de mazorcas $(\mathrm{kg})$ Peso seco promedio por planta $(\mathrm{kg}) \times 100$

Para la floración masculina se registraron los días transcurridos desde la siembra hasta la aparición del 50 $\%$ de espigas por parcela experimental, mientras que para la floración femenina se registraron los días transcurridos desde la siembra hasta la aparición de estigmas con una longitud de 0.2 a $0.3 \mathrm{~cm}$ en el $50 \%$ de las plantas de cada unidad experimental. Para determinar la altura de planta se midieron con estadal cinco plantas, elegidas al azar en cada unidad experimental, desde el suelo hasta el nudo superior. La altura de mazorca se determinó en las mismas plantas donde se midió la altura de planta, se registró la distancia en $\mathrm{cm}$ desde la base de la planta hasta el nudo con la mazorca más alta.

Se determinó la digestibilidad in vitro de la materia seca con la técnica de Tilley y Terri (1963). Para evaluar el contenido de nitrógeno se utilizó el método de Microkjeldhal (AOAC, 2012) y se multiplicó por el factor 6.25 para estimar el contenido de proteína. La fibra detergente neutro y fibra detergente ácido fueron determinadas por el método de Goering y Van Soest (1970).

\section{Análisis estadístico}

Los datos obtenidos se analizaron con los procedimientos Mixed y GLM del programa SAS para Windows, versión 8 (SAS Institute, 2001), mediante un modelo correspondiente al diseño de bloques completos al azar con el arreglo factorial descrito. La prueba de comparación de medias se realizó mediante la prueba de Tukey $(\mathrm{P} \leq 0.05)$.

\section{RESULTADOS Y DISCUSIÓN}

En el Cuadro 1 se muestran los resultados de los análisis de varianza. Se obtuvieron diferencias significativas $(P \leq 0.01)$ para el factor de variación genotipos en las variables de rendimiento de materia verde, rendimiento de materia seca, altura de planta, altura de mazorca, floración masculina, floración femenina, porcentaje de mazorca, fibra detergente neutro y fibra detergente ácido. El resto de las variables no presentó diferencias estadísticas significativas. Entre los ambientes evaluados se presentaron diferencias altamente significativas $(P \leq$ 0.01) para todas las variables, excepto para porcentaje de materia seca, que presentó diferencias significativas $(P \leq 0.05)$. Los resultados anteriores indican que la diferencia entre los ambientes evaluados impactó en el comportamiento productivo y en el valor nutricional de los diferentes híbridos.

Con respecto al factor de interacción genotipo $\times$ ambiente se encontró significancia estadísitica $(P \leq 0.01)$ en las variables rendimiento de materia verde, rendimiento de materia seca, floración femenina, porcentaje de mazorca, fibra detergente neutro, fibra detergente ácido y digestibilidad in vitro de la materia seca. Presentaron diferencias significativas $(P \leq 0.05)$ floración masculina, porcentaje de materia seca y no presentaron diferencias en altura de planta, altura de mazorca y proteína cruda.

En la prueba de comparación de medias entre genotipos (Cuadro 2), los híbridos Tlaoli Puma y H-49 AE fueron superiores $(P \leq 0.05)$ en rendimiento de materia verde (78.9 t ha-1 y $74.6 \mathrm{t} \mathrm{ha}^{-1}$ ) con respecto a los demás híbridos. El híbrido $\mathrm{H}-47$ AE presentó menor rendimiento en comparación al resto de materiales evaluados y fue inferior en 66 y $57 \%$ con respecto a Tlaoli Puma y H-49 AE, respectivamente.

El rendimiento de materia verde promedio fue de $64.5 \mathrm{t}$ $\mathrm{ha}^{-1}$, valor que se puede considerar alto en comparación con los resultados obtenidos en investigaciones realizadas por Núñez et al. (2005; 2015), quienes obtuvieron rendimientos promedio de 57.8 y $37.7 \mathrm{t} \mathrm{ha}^{-1}$, respectivamente. En investigaciones realizadas en la misma zona en que se llevó a cabo la presente evaluación, Zaragoza-Esparza et al. (2019) reportaron en híbridos de maíz rendimiento de materia verde de $64.8 \mathrm{t} \mathrm{ha}^{-1}$, valores similares a los reportados en esta investigación.

El híbrido Tlaoli Puma registró un rendimiento de materia seca de $23.1 \mathrm{t} \mathrm{ha}^{-1}$, superior $(P \leq 0.05)$ al que presentaron los híbridos H-53 AE, H 61 R, H-51 AE y H-47 AE en 35, 38, 44 y $79 \%$, respectivamente. Los híbridos H-49 AE, Atziri Puma, Tsíri Puma y CSE FESC1 tuvieron un rendimiento 
Cuadro 1. Cuadrados medios y significancia estadística para rendimiento y calidad de forraje en nueve híbridos de maíz evaluados en tres ambientes.

\begin{tabular}{|c|c|c|c|c|c|c|c|}
\hline Variable & Genotipos & Bloques & Ambientes & Gen $\times$ Amb & Error & CV (\%) & Media \\
\hline RMV & 1866.6 ** & 14.9 & $370.3 * *$ & $195.6 * *$ & 4.4 & 6.8 & 64.5 \\
\hline RMS & $77.6 * *$ & 4.9 & $58.4 \star *$ & $17.0 * *$ & 2.4 & 13.3 & 18.0 \\
\hline AP & $1480.9 * *$ & 221.9 & $6462.6 * *$ & 400.5 & 23.2 & 9.2 & 252.1 \\
\hline AM & $292.6 * *$ & 179.4 & $443.4 * *$ & 114.3 & 10.0 & 8.6 & 116.2 \\
\hline FM & $12.5 * \star$ & 4.2 & $245.4 * *$ & $5.6 *$ & 1.6 & 2.1 & 78.1 \\
\hline$F F$ & $16.4 * \star$ & 3.9 & $309.1 * *$ & $5.5 * \star$ & 1.5 & 1.9 & 79.4 \\
\hline PMca & 107.1 ** & 61.7 & $268.1 * *$ & $81.0 * \star$ & 5.5 & 13.7 & 39.8 \\
\hline PMS & 8.0 & 6.7 & $23.2 *$ & 9.5 & 2.4 & 8.5 & 28.2 \\
\hline PC & 0.9 & 0.4 & $18.7 * *$ & 0.7 & 0.9 & 10.3 & 8.6 \\
\hline FDN & $24.8 * *$ & 20.9 & $401.1 * *$ & $31.3 * *$ & 2.8 & 5.6 & 49.9 \\
\hline FDA & $21.5 * \star$ & 9.8 & $299.5 * *$ & $23.0 * *$ & 2.2 & 9.2 & 24.3 \\
\hline DIV & 25.6 & 6.5 & $137.5 * \star$ & $55.2 * \star$ & 4.0 & 5.8 & 68.1 \\
\hline
\end{tabular}

*: $P \leq 0.05, * *: P \leq 0.01, R M V$ : rendimiento de materia verde, RMS: rendimiento de materia seca, AP. altura de planta, AM: altura de mazorca, FM: floración masculina, FF: floración femenina, PMca: porcentaje de mazorca, PMS: porcentaje de materia seca, PC: proteína cruda, FDN: fibra detergente neutro, FDA: fibra detergente ácido, DIVMS: digestibilidad in vitro, CV: coeficiente de variación.

de materia seca similar a Tlaoli Puma, con una diferencia de $2.2,3.7,4.7$ y $5.4 \mathrm{t} \mathrm{ha}^{-1}$. El rendimiento de materia seca promedio fue de $18.0 \mathrm{t} \mathrm{ha}^{-1}$, valor similar al obtenido por Núñez et al. (2015) (15.8 t ha-1) y superior a los rendimientos registrados por Elizondo-Salazar (2011), con valores de

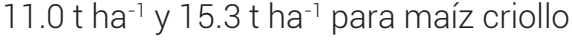

En lo que respecta al porcentaje de mazorca, es importante que el cultivo de maíz presente un alto porcentaje debido a que determina el valor energético de su ensilado (Núñez et al., 2015). Los híbridos Atziri Puma y H-47 AE presentaron un alto valor (43.7\%) y fueron superiores en $32 \%$ a H 61 R. Los demás híbridos presentaron valores similares, variando de 36 a $42 \%$, el promedio general fue de $39.8 \%$, el cual se considera un valor aceptable. Zaragoza-Esparza et al. (2019) obtuvieron valores promedio para porcentaje de mazorca semejantes a los obtenidos en la presente investigación (42 \%).

Los híbridos H-49 AE y Tlaoli Puma presentaron las plantas más altas (272 y $271 \mathrm{~cm}$, respectivamente), fueron superiores $(P \leq 0.05)$ en 17 y $16 \%$ con respecto al híbrido H-47 AE y no tuvieron diferencias con los demás híbridos evaluados. Estos materiales presentaron mayor altura que los genotipos nativos subtropicales y tropicales de Nayarit, Veracruz e Hidalgo evaluados por Rivas-Jacobo et al. (2020), quiénes registraron valores para los mejores materiales de 241.8 y $231.9 \mathrm{~cm}$, respectivamente. La altura promedio de los híbridos evaluados fue de 252 cm, superior a la registrada por Rivas et al. (2019) en 12 híbridos trilineales evaluados en Nayarit, México, que fue de $169 \mathrm{~cm}$.

En porcentaje de materia seca no se presentaron diferencias entre los diferentes genotipos evaluados ( $P$ $\leq 0.05$ ), los valores variaron entre 27.5 y $29.2 \%$, con una media de $28.2 \%$. Estos valores pueden considerarse aceptables, pues para el proceso de fermentación del ensilado no se consideran deseables valores inferiores al $25 \%$, ya que afectan el proceso de fermentación por el alto contenido de agua, mientras que valores de materia seca superiores a $35 \%$ dificultan la compactación del material por el bajo contenido de agua, lo que provoca que no se elimine totalmente el oxígeno y, en consecuencia, se presenten fermentaciones pútridas de tipo butírico, que disminuyen la calidad del ensilado (Zardin et al., 2017).

Para el contenido de proteína cruda no existieron diferencias significativas $(P \leq 0.05)$ entre híbridos, los valores variaron entre 8.1 y $9.1 \%$ y presentaron una media general de $8.6 \%$. Estos valores son mayores a los reportados por Núñez et al. (2015) en cuatro híbridos de maíz de la empresa Pioneer en la Región Lagunera estado de Coahuila, México, que variaron entre 6.8 y $8.2 \%$, con una media de $7.2 \%$.

Para fibra detergente neutro, el híbrido H 61 R presentó el valor más bajo (47.5\%), inferior en $10 \%$ con respecto a 
Cuadro 2. Comparación de medias entre nueve híbridos de maíz de Valles Altos de México considerando la media de tres ambientes. Ciclo primavera-verano 2016.

\begin{tabular}{|c|c|c|c|c|c|c|c|}
\hline \multirow{2}{*}{ Genotipo } & RMV & RMS & \multirow{2}{*}{$\begin{array}{c}\text { PMca } \\
\%)\end{array}$} & FM & $\mathrm{FF}$ & AP & AM \\
\hline & \multicolumn{2}{|c|}{$\left(\right.$ t ha-1 $\left.^{-1}\right)$} & & \multicolumn{2}{|c|}{ (días) } & \multicolumn{2}{|c|}{$(\mathrm{cm})$} \\
\hline Tlaoli Puma & $78.9 a$ & $23.1 \mathrm{a}$ & $41.8 \mathrm{a}$ & $77 b$ & $78 b$ & $271 \mathrm{a}$ & $114 a$ \\
\hline $\mathrm{H}-49 \mathrm{AE}$ & $74.6 \mathrm{ab}$ & $21 a b$ & $38.6 a b$ & $77 \mathrm{~b}$ & $78 b$ & $273 a$ & $125 a$ \\
\hline CSE FESC 1 & 70.8 bc & $17.7 \mathrm{bc}$ & $40.4 a b$ & $78 b$ & $80 \mathrm{~b}$ & $255 a b$ & $112 a$ \\
\hline Atziri Puma & $68.8 \mathrm{bc}$ & 19.4 ac & $43.6 \mathrm{a}$ & $78 \mathrm{~b}$ & $79 b$ & $251 \mathrm{ab}$ & $120 a$ \\
\hline Tsíri Puma & $64.7 \mathrm{dc}$ & $18.4 \mathrm{bc}$ & $35.8 a b$ & $78 b$ & $79 b$ & $247 a b$ & $111 a$ \\
\hline $\mathrm{H}-53 \mathrm{AE}$ & $60.2 \mathrm{de}$ & $17.1 \mathrm{c}$ & $40.6 a b$ & $81 \mathrm{a}$ & $83 a$ & $249 a b$ & $118 a$ \\
\hline H61 R & $57.7 \mathrm{e}$ & $16.7 \mathrm{c}$ & $33.2 b$ & $77 \mathrm{~b}$ & $79 b$ & $243 a b$ & $113 a$ \\
\hline $\mathrm{H}-51 \mathrm{AE}$ & $57.6 \mathrm{e}$ & $16 \mathrm{~cd}$ & $40.7 a b$ & $78 b$ & $79 b$ & $248 a b$ & $124 a$ \\
\hline $\mathrm{H}-47 \mathrm{AE}$ & $47.5 f$ & $12.9 \mathrm{~d}$ & $43.7 \mathrm{a}$ & $78 b$ & $80 \mathrm{~b}$ & $233 b$ & $110 a$ \\
\hline D.S.H. (0.05) & 6.8 & 3.7 & 8.5 & 2.4 & 2.4 & 35.4 & 15.3 \\
\hline
\end{tabular}

Medias con letras iquales en las columnas no son estadísticamente diferentes (Tukey, $\mathrm{P} \leq 0.05$ ). RMV: rendimiento de materia verde, RMS: rendimiento de materia seca, PMca: porcentaje de mazorca, FM: floración masculina, FF: floración femenina, AP. altura de planta, AM: altura de mazorca.

Atziri Puma, los demás híbridos no mostraron diferencias $(P \leq 0.05)$, con valores entre 48.0 y $52.4 \%$ y una media de $49.9 \%$. Juráček et al. (2012) registraron para fibra detergente neutro valores de $45.9 \%$ en maíces de la región oriental de Eslovaquia. Para fibra detergente ácido tampoco se encontraron diferencias significativas $(P \leq$ 0.05 ) entre híbridos. La media obtenida fue de $24.3 \%$, valor similar al reportado por Juráček et al. (2012) de 24.7\% en maíces de Europa oriental y a los obtenidos por Núñez et al. (2015) en híbridos de una empresa evaluados en la región Lagunera, quienes registraron una media de $25 \%$ y una variación de 16.6 a $30.9 \%$. Los valores de fibras detergente neutro y fibra detergente ácido no presentaron diferencias entre los híbridos evaluados; sin embargo, éstos fueron bajos, lo que es una característica deseable en el forraje, ya que permite un mayor aprovechamiento del alimento por parte del animal.

Para digestibilidad in vitro de la materia seca no se encontraron diferencias significativas $(P>0.05)$, la media obtenida para esta variable fue de $68.1 \%$, por lo que se considera un valor alto si lo se compara con el registrado por Sánchez e Hidalgo-Ardón (2018), quiénes obtuvieron porcentajes que variaron de 60.6 a $65.6 \%$ en nueve híbridos

Cuadro 3. Comparación de medias de variables de valor nutricional en nueve híbridos de maíz evaluados en tres ambientes de Valles Altos de México. Ciclo primavera - verano 2016.

\begin{tabular}{lccccc}
\hline \multirow{2}{*}{ Genotipo } & MS & PC & FDN & FDA & DIV \\
\cline { 2 - 6 } & \multicolumn{5}{c}{$\%$} \\
H-49 AE & $28.1 \mathrm{a}$ & $8.4 \mathrm{a}$ & $48.0 \mathrm{ab}$ & $22.3 \mathrm{~b}$ & $69.8 \mathrm{a}$ \\
CSE FESC 1 & $26.9 \mathrm{a}$ & $8.7 \mathrm{a}$ & $48.2 \mathrm{ab}$ & $23.1 \mathrm{ab}$ & $68.7 \mathrm{a}$ \\
Atziri Puma & $28.0 \mathrm{a}$ & $8.4 \mathrm{a}$ & $52.4 \mathrm{a}$ & $26.6 \mathrm{a}$ & $68.5 \mathrm{a}$ \\
Tlaoli Puma & $29.2 \mathrm{a}$ & $8.1 \mathrm{a}$ & $51.4 \mathrm{ab}$ & $25.3 \mathrm{ab}$ & $67.0 \mathrm{a}$ \\
Tsíri Puma & $29.5 \mathrm{a}$ & $8.4 \mathrm{a}$ & $50.5 \mathrm{ab}$ & $24.8 \mathrm{ab}$ & $69.3 \mathrm{a}$ \\
H-53 AE & $28.4 \mathrm{a}$ & $8.6 \mathrm{a}$ & $50.6 \mathrm{ab}$ & $25.3 \mathrm{ab}$ & $66.1 \mathrm{a}$ \\
H-51 AE & $27.7 \mathrm{a}$ & $9.1 \mathrm{a}$ & $50.1 \mathrm{ab}$ & $24.1 \mathrm{ab}$ & $67.7 \mathrm{a}$ \\
H61 R & $28.7 \mathrm{a}$ & $8.8 \mathrm{a}$ & $47.5 \mathrm{~b}$ & $22.0 \mathrm{~b}$ & $70.5 \mathrm{a}$ \\
H-47 AE & $27.5 \mathrm{a}$ & $8.9 \mathrm{a}$ & $50.9 \mathrm{ab}$ & $25.3 \mathrm{ab}$ & $65.5 \mathrm{a}$ \\
DSH (0.05) & 3.7 & 1.4 & 4.4 & 3.5 & 6.2 \\
\hline
\end{tabular}

Medias con letras iguales en las columnas no son estadísticamente diferentes (Tukey, P $\leq 0.05)$. MS: materia seca, PC: proteína cruda, FDN: fibra detergente neutro, FDA: fibra detergente ácido, DIV: digestibilidad in vitro, DSH: diferencia significativa honesta. 
de maíz del CIMMYT evaluados en la zona alta lechera de Cartago, Costa Rica. La mayor o menor digestibilidad in vitro de la materia seca en un ensilado es resultado de la digestibilidad de la materia seca y de los contenidos de fibra detergente neutro y fibra detergente ácido.

A mayor digestibilidad se reduce el contenido de fibra detergente neutro y el de fibra detergente ácido y se incrementa la disponibilidad de nutrimentos a nivel ruminal del ensilado; además, se incrementa el consumo de alimento, se maximiza la síntesis de proteína microbiana y, en consecuencia, se obtiene mayor producción animal, ya sea en leche o ganancia de peso (Núñez et al., 2015).

Al realizar la prueba de comparación de medias en los tres ambientes evaluados (Cuadro 4), se encontró en la FES Cuautitlán, UNAM un rendimiento de materia verde superior $(P \leq 0.05)$ en 7.0 y $12.1 \%$ a los rendimientos obtenidos en CEVAMEX tanto en la primera como en la segunda fecha de cosecha. Estos resultados pueden deberse a las condiciones climáticas de los dos ambientes, en FES Cuautitlán, UNAM la siembra se llevó a cabo el 6 de junio, pocos días antes de que iniciara el máximo periodo de precipitación del año, lo que favoreció el rápido desarrollo del cultivo, mientras que en CEVAMEX la siembra se realizó el 25 de mayo, cuando faltaban 33 días para el inicio del máximo periodo de precipitación en esa localidad, lo que pudo establecerse como una limitante para el desarrollo inicial del cultivo.

El rendimiento de materia seca fue superior $(P \leq 0.05)$ en el ambiente de la FES Cuautitlán, UNAM con respecto al obtenido en las dos fechas de cosecha en CEVAMEX, en 11.3 y $17.3 \%$, para la primera y segunda fecha de cosecha, respectivamente.

Para las floraciones femenina y masculina se encontraron diferencias entre ambientes $(P \leq 0.05)$, lo mismo ocurrió para altura de planta y altura de mazorca. El porcentaje de mazorca obtenido en la FES Cuautitlán, UNAM fue superior $(P \leq 0.05)$ en 10.3 y $24.2 \%$ con respecto a los registrados en CEVAMEX1 y CEVAMEX2. Los porcentajes de mazorca variaron de 36.3 a $45.1 \%$ y fueron menores a los registrados en la Comarca Lagunera por Núñez et al. (2015) de $37.80 \%$ a $57.69 \%$ con una media de $48.6 \%$.

La prueba de comparación de medias entre ambientes para las variables de valor nutricional (Cuadro 5) evidenció diferencias significativas $(P \leq 0.05)$ en porcentaje de materia seca, proteína cruda, fibra detergente neutro y fibra detergente ácido. En FES Cuautitlán, UNAM se presentó un porcentaje de materia seca superior $(P \leq 0.05)$ en $15 \%$ con respecto a CEVAMEX1. Este resultado se relaciona positivamente con los resultados obtenidos para rendimiento de materia seca.

El porcentaje de proteína cruda en CEVAMEX1, a los 134 días de crecimiento, fue mayor $(P \leq 0.05)$ con respecto a CEVAMEX2 y a la FES Cuautitlán, UNAM; lo anterior pudo ser consecuencia de que para esta fecha el cultivo permaneció un mayor número de días en campo (ocho) con respecto a la FES Cuautitlán, UNAM, permitiendo un mayor desarrollo del grano $y$, en consecuencia, mayor acumulación de proteína; en contraste, en CEVAMEX el contenido de proteína cruda disminuyó en $0.7 \%$ en la segunda fecha de cosecha con respecto a la primera, lo que puede atribuirse a que a los 134 días el cultivo alcanzó la madurez óptima para cosecha, lo que provocó mayores contenidos de proteína, mientras que a los 148 días empezaron a disminuir, lo que incrementó el contenido estructural de la planta, limitando su disponibilidad. En estudios realizados por Núñez et al. (2015) en híbridos en la región Lagunera se obtuvieron valores de proteína de 6.8 a $8.2 \%$, los cuales son inferiores a los obtenidos en esta investigación (7.7 a $9.4 \%$ ).

En CEVAMEX1 se obtuvieron valores más bajos para fibra detergente neutro y para fibra detergente ácido, menores en 15.8 y $30.0 \%$ con respecto a CEVAMEX2 y menores en $4.3 \%$ y $9.3 \%$ con respecto a FES Cuautitlán, UNAM. Estos valores bajos de fibras se asociaron con una mayor digestibilidad in vitro de la materia seca. Los valores de fibra detergente neutro y fibra detergente ácido obtenidos en la presente investigación variaron de 46.8 a $54.2 \%$ en fibra detergente neutro y de 21.5 a $28.0 \%$ en fibra detergente ácido, los cuales se consideran dentro de los intervalos que presenta un ensilado de maíz de alta calidad forrajera. Al respecto, Núñez et al. (2015) indicaron que el valor óptimo de fibra detergente neutro en un ensilado de maíz debe ser menor de $50 \%$, mientras que el contenido de fibra detergente ácido debe ser en promedio de $26 \%$ (Kolver et al., 2001).

La comparación de medias para la variable digestibilidad in vitro de la materia seca evidenció diferencias significativas $(P \leq 0.05)$ en la FES Cuautitlán, UNAM y CEVAMEX1 (69.6 y $69.3 \%$ ), con respecto a CEVAMEX2 $(65.5 \%)$, coincidiendo este comportamiento con el mayor porcentaje de mazorca, factor asociado comúnmente con la digestibilidad (Núñez, 2003) en 18 híbridos comerciales de maíz en la región Lagunera del estado de Coahuila, México. Lo anterior es consecuencia de la mayor madurez a la que fueron cosechados los híbridos de maíz, ya que conforme incrementó la madurez se redujo la digestibilidad. Estos resultados fueron similares a los obtenidos por Lynch et al. (2013) en los cultivares de maíz Tassilo, Andante y KXA 7211 evaluados en Irlanda, quienes reportaron menor digestibilidad con cosechas tardías. 
Cuadro 4. Comparación de medias en tres ambientes considerando la media de nueve híbridos evaluados. Ciclo primaveraverano 2016.

\begin{tabular}{|c|c|c|c|c|c|c|c|}
\hline \multirow{2}{*}{ Ambiente } & RMV & RMS) & FM & FF & AP & AM & \multirow{2}{*}{$\begin{array}{c}\text { Mazorca } \\
\%\end{array}$} \\
\hline & \multicolumn{2}{|c|}{$\left(\mathrm{t} \mathrm{ha}^{-1}\right)$} & \multicolumn{2}{|c|}{ (días) } & \multicolumn{2}{|c|}{$(\mathrm{cm})$} & \\
\hline FES Cuautitlán, UNAM & $68.5 \mathrm{a}$ & $19.7 \mathrm{a}$ & $81.2 \mathrm{a}$ & $83 a$ & $249.7 a$ & $113 a$ & $45.1 \mathrm{a}$ \\
\hline CEVAMEX1 & $64 \mathrm{~b}$ & $17.7 \mathrm{~b}$ & $77.7 \mathrm{~b}$ & $78.8 b$ & $251.4 \mathrm{a}$ & $117 a$ & $40.9 \mathrm{~b}$ \\
\hline CEVAMEX2 & $61.1 \mathrm{~b}$ & $16.8 b$ & $75.2 \mathrm{c}$ & $76.3 \mathrm{c}$ & $255.3 \mathrm{a}$ & $118 a$ & $36.3 c$ \\
\hline DSH (0.05) & 2.9 & 1.6 & 1.0 & 1.0 & 15.2 & 6.6 & 3.6 \\
\hline
\end{tabular}

Medias con letras iguales en las columnas no son estadísticamente diferentes (Tukey, $\mathrm{P} \leq 0.05$ ). RMV: rendimiento de materia verde, RMS: rendimiento de materia seca, FM: floración masculina, FF: floración femenina, AP. altura de planta, AM: altura de mazorca, DSH: diferencia significativa honesta.

Cuadro 5. Comparación de medias de nueve híbridos para las variables de calidad de forraje evaluados en tres ambientes. Ciclo primavera-verano 2016.

\begin{tabular}{lccccc}
\hline \multirow{2}{*}{ Ambiente } & MS & PC & FDN & FDA & DIV \\
\cline { 2 - 5 } & & & $\%$ & & \\
\hline FES Cuautitlán, & $29.2 \mathrm{a}$ & $7.7 \mathrm{c}$ & $48.8 \mathrm{~b}$ & $23.5 \mathrm{~b}$ & $69.6 \mathrm{a}$ \\
UNAM & $27.9 \mathrm{ab}$ & $9.4 \mathrm{a}$ & $46.8 \mathrm{c}$ & $21.5 \mathrm{c}$ & $69.3 \mathrm{a}$ \\
CEVAMEX1 & $27.4 \mathrm{~b}$ & $8.7 \mathrm{~b}$ & $54.2 \mathrm{a}$ & $28 \mathrm{a}$ & $65.5 \mathrm{~b}$ \\
CEVAMEX2 & 1.6 & 0.6 & 1.9 & 1.5 & 2.6 \\
DSH (0.05) & &
\end{tabular}

Medias con letras iguales en las columnas no son estadísticamente diferentes (Tukey, P $\leq 0.05)$. MS: materia seca, PC: proteína cruda, FDN: fibra detergente neutro, FDA: fibra detergente ácido, DIV: digestibilidad in vitro, DSH: diferencia significativa honesta.

\section{CONCLUSIONES}

Los híbridos Tlaoli Puma y H-49 AE presentaron mayor productividad y calidad, constituyendo una alternativa para los productores de ganado de los Valles Altos de México. Los híbridos bajo condiciones de punta de riego deben ser cosechados a los 125 o 126 días para obtener mayores rendimientos de forraje, tanto en materia seca como en materia verde, un mayor porcentaje de mazorca y menores contenidos de fibra detergente ácido, características que impactan sobre la calidad de un ensilado, lo cual se vería reflejado en la producción del ganado.

\section{AGRADECIMIENTOS}

El presente trabajo se llevó a cabo con financiamiento del Programa de Apoyo a Proyectos de Investigación e Innovación Tecnológica (PAPIIT) clave: IT201618, de la Dirección General de Asuntos del Personal Académico (DGAPA), UNAM.

\section{BIBLIOGRAFÍA}

AOAC, Association of Analytical Chemists (2012) Official Methods of Analysis of AOAC International. Vol. 1. 19th edition. Association of Analytical Chemists. Washington, D.C. USA. 672 p.
Elizondo-Salazar J. A. (2011) Influencia de la variedad y altura de cosecha sobre el rendimiento y valor nutritivo de maíz para ensilaje. Agronomía Costarricense 35:105-111, https://doi org/10.15517/RAC.V35I2.6683

Goering H. and P. Van Soest (1970) Forage Fiber Analysis (Apparatus, Reagents, Procedures and Some Applications). Agricultural Handbook No. 379. Agricultural Research Service, United States Department of Agriculture. Washington, DC. 76 p.

González C. F., A. Peña R., G. Núñez H. y C. A. Jiménez G. (2005) Efecto de la densidad y altura de corte en el rendimiento y calidad del forraje de maíz. Revista Fitotecnia Mexicana 28:393-397.

Grant J. R. and A. Adesogant (2018) Journal of Dairy Science Silage Special Issue: Introduction. Journal of Dairy Science 101:39353936, https://doi.org/10.3168/jds.2018-14630

Juráček M., D. Bíro, M. Šimko, B. Gálik and M. Rolinec (2012) The quality of maize silages from west Region of Slovakia. Journal of Central European Agriculture 13:695-703, https:/doi.org/10.5513/ JCEA01/13.4.1114

Kolver E. S., J. R. Roche, D. Miller and R. Densley (2001) Maize silage for dairy cows. Proceedings of the New Zealand Grassland Association 63:195.201, https://doi.org/10.33584/jnzg.2001.63.2407

Lynch J. P., P. O'Kiely and E. M. Doyle (2013) Yield, nutritive value and ensilage characteristics of whole-crop maize, and of the separated cob and stover components - nitrogen, harvest date and cultivar effects. The Journal of Agricultural Science 151:347-367, https://doi.org/10.1017/S0021859612000342

Martin N. P., M. P. Russelle, J. M. Powell, C. J. Sniffen, S. I. Smith, J. M. Tricarico and R. J. Grant (2017) Sustainable forage and grain crop production for the US dairy industry. Journal of Dairy Science 100:9479-9494, https://doi.org/10.3168/jds.2017-13080

Núñez H. G., A. Anaya S., R. Faz C. y H. A. Serrato M. (2015) Híbridos de maíz forrajero con alto potencial de producción de leche de bovino. Agrofaz 15:47-56.

Núñez H. G., C. Faz R., F. González C. y A. Peña R. (2005) Madurez de 
híbridos de maíz a la cosecha para mejorar la producción y calidad del forraje. Técnica Pecuaria en México 43:69-78.

Núñez H. G, E. Contreras G. y C. Faz R. (2003) Características agronómicas y químicas importantes en híbridos de maíz para forraje con alto valor energético. Técnica Pecuaria en México 41:37-48.

Rivas J. M. A., A. Carballo C., A. R. Quero C., A. Hernández G., H. Vaquera H., E. C. Rivas Z., ... y E. J. Rivas Z. (2018) Comportamiento productivo de doce híbridos trilineales de maíz para forraje en una región tropical seca. Tropical and Subtropical Agroecosystems 21:579-586

Rivas-Jacobo M., G. Ballesteros-Rodea, R. Lepe-Aguilar, A. Zaragoza-Bastida, C. Ibarra-Gudiño y N. Rivero-Pérez (2020) Respuesta productiva de maíces subtropicales y tropicales con fines forrajeros en una región semiárida. Abanico Agroforestal 2:1-13, https://doi. org/10.37114/abaagrof/2020.6

Sánchez L. W. y C. Hidalgo-Ardón (2018) Potencial de forraje de nueve híbridos de maíz en la zona alta lechera de Costa Rica. Agronomía Mesoamericana 29:153-164, https://doi. org/10.15517/ma.v29i1.27732
SAS Institute (2001) SAS User's Guide. Release 8.1. 6th edition. SAS Institute, Inc. Cary, North Carolina, USA. 956 p.

Tilley J. M. A. and R. A. Terry (1963) A two stage technique for the in vitro digestion of forage crops. Grass and Forage Science 18:104111, https://doi.org/10.1111/j.1365-2494.1963.tb00335.x

Zaragoza-Esparza J., M. Tadeo-Robledo, A. Espinosa-Calderón, C. LópezLópez, J. C. García-Espinosa, B. Zamudio-González, ... y F. RosadoNúñez (2019) Rendimiento y calidad de forraje de híbridos de maíz en Valles Altos de México. Revista Mexicana de Ciencias Agrícolas 10:101-111, https://doi.org/10.29312/remexca. v10i1.1403

Zardin P. B., J. P. Velho, C. C. Jobim, D. R. M. Alessio, I. M. P. Haygert-Velho, G. M. da Conceição, and P. S. G. Almeida (2017) Chemical composition of corn silage produced by scientific studies in Brazil - A metaanalysis. Semina: Ciências Agrárias 38:503-512, https://doi. org/10.5433/1679-0359.2017v38n1p503 